\title{
„Taub im Kopf?“ - Chancen und Risiken in der Entwicklung von hörenden Kindern gehörloser Eltern
}

\section{“Deaf in the Head?": Chances and Risks in the Development of Hearing Children of Deaf Parents}

Hiltrud Funk

\author{
Hörende Kinder von gehörlosen Eltern (Codas) leben im Spannungsfeld von \\ unterschiedlichen kulturellen Umgebungen. Sprachliche und psychosoziale Aus- \\ wirkungen der Familiensituation sind besonders von Interesse für die Forschung. \\ Überwiegend wird die Situation trotz teilweise beträchtlicher Belastungen \\ gemeistert. Insoweit es zu Beeinträchtigungen in der Entwicklung kommt bzw. \\ diese drohen, bedarf es spezialisierter professioneller Unterstützungssysteme.
}

\section{LERNZIEL}

Ziel ist, die besondere Situation von hörenden Kindern gehörloser Eltern übersichtartig kennenzulernen. Die Vielfalt der Entwicklungen im Spannungsfeld von unterschiedlichen Lebenserfahrungen soll deutlich werden. Schwerpunkte liegen in der Sprachentwicklung und der psychosozialen Entwicklung.

\section{Einleitung}

Die deutschsprachige Forschung beschäftigt sich nur wenig mit der besonderen Situation von hörenden Kindern und deren gehörlosen Eltern. Kinder von gehörlosen Eltern sind zu 90-95\% hörend. In den letzten Jahren hat sich auch in Deutschland für hörende Kinder gehörloser Eltern der Begriff Coda (children of deaf adults) durchgesetzt. Erste wissenschaftliche Veröffentlichungen drehen sich um die Frage der Lautsprachentwicklung [1]. Größere Arbeiten in der Folgezeit mit den Themen frühkindliche Entwicklung, Lautsprachentwicklung, Prävention und bimodal-bilinguale Sprachentwicklung sind in zeitlicher Reihenfolge des Erscheinens von Funk [2] Grüner [3)] Peter/Raith-Kaudelka/Scheithauer [4] und aktuell Chilla/Hofmann [5] veröffentlicht.

Die englischsprachige Forschung kann auf den Beginn in den 70er/80er-Jahren des letzten Jahrhunderts zurückblicken. Auch hier liegt ein deutlicher Schwerpunkt auf der Sprachentwicklung, wurde jedoch z.B. durch die Werke von Preston [6], Meadow-Orlans/Spencer/ Koester [7] und Bishop/Hicks [8] auf die Bereiche aus- geweitet, die auch diesen Artikel bestimmen. Viele Aussagen zu Codas und ihren Eltern beziehen sich auf die amerikanische Forschung, sind deshalb nicht so ohne Weiteres auf deutsche Verhältnisse anzuwenden. Einschränkend ist zu vermerken, dass die Untersuchungsgruppen oft klein sind bzw. das Bildungsniveau der Untersuchungsteilnehmer/innen einseitig hoch ist.

\section{Ambiguität und Identitätsarbeit}

Eine Coda äußerte auf die Frage nach möglichen Problemen: „Der schreckliche Moment, wenn du winkst und auf den Boden stampfst um Aufmerksamkeit zu bekommen, und du bemerkst, dass du von Hörenden umgeben bist." Offensichtlich peinlich für die junge Frau und vermutlich auf Unverständnis und Irritation stoßend bei den Hörenden schildert sie das Zusammentreffen von Verhaltensweisen, die in unterschiedlichen Kontexten erlernt wurden. Die Notwendigkeit, die Aufmerksamkeit der gehörlosen Eltern durch visuelle Zugehensweisen zu gewinnen, prallt auf die für ein hörendes Umfeld geschaffene, mehr auditiv orientierte und scheinbar „diskreter“ agierende Art. Missverständnisse zwischen hörendem Umfeld und Codas sind vorprogrammiert. So wird z. B. eine bestimmte Mimik, in der wh-Fragewörter (z.B. who, where) in American Sign Language (ASL) produziert werden, als Ausdruck von negativen Affekten fehlgedeutet. Familien mit hörenden Kindern und gehörlosen Eltern leben in einem Spannungsfeld zwischen Normen und Werten, die jeweils in einer hörenden und einer gehörlosen Umgebung gelten. Gehörlose Menschen sehen sich in der Regel als nicht behindert, während das hörende Umfeld ihr So-Sein nicht nur als unterschiedlich, sondern als behindert betrachtet. Die Gebärdensprache als ihr Kommunikationsmittel in der Familie und in der Gehör- 
losengemeinschaft, von ihnen als alltäglich von Beginn an erlebt, erfährt in der Gesellschaft eine besondere Beachtung. Sie fällt auf, markiert einen Unterschied zur Sprache der hörenden Mehrheitsgesellschaft und wird teilweise immer noch nicht als „richtige“ Sprache gesehen. Codas leben diesen Spagat. Sie sind besonders dadurch, dass ihre Eltern anders sind.

Merke

Hören und Sprechen markieren die Unterschiede zu den Eltern und bedeuten gleichzeitig eine Ressource für die Kinder. Die Realisierung der Differenzen zu den Eltern ist ein Prozess, der sehr individuell und je nach Umfeld geleistet wird; oft wird er durch den Besuch des Kindergartens und der Schule ausgelöst.

Codas beschreiben ihre Erfahrungen als Außenseiter, als Fremde, als Mittelsmänner und -frauen oder als jemand, der gleichsam an eine gläserne Decke stößt. Sie betrachten sich als Gruppe mit eigener Identität jenseits der Zugehörigkeit zur hörenden Mehrheit und gehörlosen Minderheit. Sie erleben somit vermehrt Konflikte zwischen hörenden und gehörlosen Werten, Normen und Verhaltensweisen [9].

\section{Sprachentwicklung}

\section{Lautsprach- und Gebärdensprachent- wicklung}

FRAGLICHE AUSGANGSLAGE

„Wie hast du sprechen gelernt?“ Mit dieser Frage werden Codas oft konfrontiert. Die Fragenden gehen von ihren meist rudimentären Erfahrungen mit gehörlosen Menschen aus und sind überrascht über das Ausmaß der Sprachkompetenz von Codas.

Tatsächlich beschäftigte sich die Forschung zunächst mit der Klärung dieser Frage. Im frühen Säuglingsalter ist die Kommunikation zwischen Mutter $^{1}$ und Kind noch nicht von Sprache dominiert. Mit steigendem Alter des Kindes erhält jedoch die sprachliche Kommunikation auch für die Beziehung und die psychosoziale sowie kognitive Entwicklung eine große Rolle. Missverständnisse zwischen gehörloser Mutter und hörendem Kind werden sichtbar, da die lautlichen Produktionen des Kindes von der Mutter abgesehen werden müssen und dies nicht immer gelingen kann. Der elterliche Input ist sehr individuell. Eltern gebrauchen die Lautsprache und die Gebärdensprache, und zwar separat, gemischt oder simultan. Sowohl Lautsprache als auch Ge-

\footnotetext{
1 Mutter steht hier für Menschen, die mütterliche Funktionen erfüllen.
}

bärdensprache werden normgerecht oder mehr oder minder abweichend eingesetzt.

Die Ergebnisse der Forschung zur Klärung des Niveaus der Lautsprache von jungen Codas sind im Einzelnen nicht unbedingt übereinstimmend. Englischsprachige Untersuchungen belegen überwiegend, dass eine große Anzahl von Codas Verzögerungen in der Lautsprachentwicklung zeigen. Schiff/Ventry kommen zu dem Ergebnis, dass $44 \%$ der Codas Probleme im Sprechen oder der Sprache oder beidem haben [10].

Grüners Studie mit 117 Kindern zeigt, dass Codas im Alter von 3 und 6 Jahren hinsichtlich einer Normgruppe bei der Lautunterscheidung nicht unterschiedlich sind. Die Lautbildung bewegt sich unterhalb der Norm. Mit wachsendem Alter nehmen die Auffälligkeiten jedoch ab und der allgemeine Sprachstand ist unauffällig. Positiv die Lautsprache beeinflussen die Artikulationsfähigkeit und die Lautsprachkompetenz der Eltern, der häufigere Kontakt zu hörenden Menschen und Einzelkind oder Erstgeborener zu sein. Der Gebrauch der Gebärdensprache verbessert den Wortschatz der Kinder, verschlechtert allerdings die Grammatik. Da es sich hier um ein Aufwachsen in Bilingualität handelt, ist zu erwarten, dass von einem Übergangsphänomen auszugehen ist [3].

Die Pilotstudie von Chilla/Hofmann mit sechs Codas im Alter von 3,10 bis 6,2 Jahren statuiert für 3 Kinder vergleichbare Fähigkeiten wie bei hörenden monolingualen Kindern. Große Abweichungen finden sich jedoch bei den übrigen Kindern hinsichtlich des produktiven Wortschatzes von Verben, der Präpositionen und der Morphosyntax. Damit wäre die Hälfte der untersuchten Kinder sprachgestört. Die Autorinnen legen jedoch in der Folge Wert auf die Untersuchung der bimodalen Bilingualität, deren Erforschung in Deutschland gerade erst beginnt [5].

\section{Merke}

Die Untersuchungen zur Lautsprachentwicklung von Codas zeigen sehr unterschiedliche Ergebnisse. Sie reichen von keiner Gefährdung bis zu 50 \% Sprachstörungen.

Es existieren kaum Untersuchungen, die sich ausschließlich mit der Gebärdensprachentwicklung von Codas befassen. Man hat jedoch gefunden, dass Codas im Babyalter, mit denen in Gebärdensprache kommuniziert wird, eine Lallphase durchlaufen, die an dem Gebärdensprachinput orientiert ist. Sie reduzieren die spezifische rhythmische Frequenz ihrer Handbewegungen dann, wenn sie eine linguistische Aktivität produzieren [11]. Unterschiedliche Aussagen gibt es zum Zeitpunkt des ersten Wortes bzw. der ersten Gebärde. Meistens wird von einem mit der Lautsprache ver- 
gleichbaren Beginn ausgegangen. Es gibt jedoch auch Hinweise auf ein früheres Erscheinen der ersten Gebärde [12].

\section{Merke}

Codas im Babyalter lallen gleichsam mit ihren Händen. Über den Zeitpunkt des Erscheinens der ersten Gebärde herrscht Uneinigkeit.

\section{Bimodale Bilingualität}

Der sprachlichen Situation von Codas umfassender gerecht wird der Ansatz der bimodalen Bilingualität. Der Erwerb von Lautsprache und Gebärdensprache im Sinne von Bilingualität unterscheidet sich grundlegend vom gleichzeitigen Erwerb von 2 Lautsprachen. Bilinguale Menschen mit 2 Lautsprachen werden diese immer nacheinander sprechen müssen. Codeswitching bezeichnet den Wechsel zwischen den beiden Sprachen. Bilinguale Menschen jedoch, die gleichzeitig mit Lautsprache und Gebärdensprache aufwachsen bzw. diese erwerben, können simultan Gebärden und Sprechen nutzen. Gebärdensprache wird vorwiegend visuell erfasst. Die Lautsprache wird im Wesentlichen auditiv aufgenommen. Beide Systeme nutzen unterschiedliche Modi der Sprachproduktion und -perzeption.

\footnotetext{
Merke

Codas wachsen in ihrem Elternhaus in unterschiedlichem Maß in Gebärdensprache auf und haben gleichzeitig Zugang zur gesprochenen Sprache, sie sind bimodal-bilingual.
}

Auch in diesem Untersuchungsgebiet sind die Ergebnisse nicht einheitlich. So finden Jones/Quigley, dass Frageformen der beiden Sprachen bei Codas völlig separat erworben werden und nicht interferieren [13]. Andere Forscher dagegen stellen sehr wohl eine Beeinflussung der Lautsprache durch die Gebärdensprache fest. Diese zeigt sich z. B. stark bei den Frageformen, beim Auslassen von Artikeln oder einer Grammatik, die der Regel der ASL folgt [14]. Hier kommt spätestens die Frage auf, wie diese Auffälligkeiten interpretiert werden: ob als Fehler, weil nicht der Grammatik der Lautsprache entsprechend oder als Ausdruck der bimodalen Bilingualität.

Chilla/Hofmann setzen zur Untersuchung der bimodaIen Bilingualität das „Hamburger Verfahren zur Analyse des Sprachstandes bei Fünfjährigen“ ein - ein Screening für mehrsprachig aufwachsende Kinder und zudem jetzt für die Deutsche Gebärdensprache (DGS) adaptiert. Die Ergebnisse zeigen, dass alle Kinder die Satzstruktur der Lautsprache erwerben und die Verbkongruenz in der DGS gelungen ist. Allerdings finden sich nur wenige flektierte Verben in der DGS. Die DGS wird demnach nicht umfassend erworben [5].
Die bimodale bilinguale Sprachentwicklung und der Sprachgebrauch von Codas sind maßgeblich von der Sprachwahl und der Kompetenz der Eltern bestimmt. Die Kinder passen sich aktiv und kreativ sehr früh in ihrem Verhalten, auch dem sprachlichen, den Bedingungen der Gehörlosigkeit ihrer Eltern an und umgekehrt die Eltern dem Hören der Kinder. Sowohl Mütter als auch Kinder produzieren Äußerungen im Codeblending, d. h. sie gebrauchen simultan Gebärden und Worte mit und ohne Stimme.

Mit steigendem Alter der Kinder nimmt Codeblending bei den Kindern zu. Lautsprache hat mit 3 Jahren den höchsten Anteil, die Gebärdensprache den geringsten. Die Mütter gebrauchen mit steigendem Alter der Kinder mehr Gebärdensprache. Der Anteil an Codeblending bleibt über die Zeit bei fast $60 \%$. Außerdem produzieren die hörenden Kinder längere Äußerungen als ihre gehörlosen Mütter. Das in anderen Untersuchungen gefundene, die Gebärdensprache begleitende Flüstern der Codas, wenn sie mit gehörlosen Menschen oder mit anderen Codas kommunizieren, wird auch hier beobachtet [15].

Merke

Codeblending wird durch die simultane Verwendung von Worten und Gebärden gebildet.

Die durchweg zu beobachtende Bevorzugung des Codeblendings durch Codas, auch der erwachsenen, in der Kommunikation mit gehörlosen Menschen erklärt sich dadurch, dass der simultane Gebrauch von Lautsprache und Gebärdensprache keine Verluste bringt. Im Gegenteil wird das Verstehen verbessert. Codeblending fördert die Integration von lexikalischem Material aus beiden Sprachen und die Semantik. Beide Sprachen werden im Gehirn gleichzeitig aktiviert, wie neurolinguistische Forschung zeigt. Unimodal bilinguale Menschen hingegen müssen eine Sprache unterdrücken, um zwischen den Sprachen zu switchen bzw. um zu verstehen, was den Prozess verlangsamt [16].

\section{Merke \\ Codeblending erleichtert das Verstehen. \\ Das Hören und die auditive Verarbeitung und Wahrnehmung}

Per Definition müssen die hörenden Kinder von gehörlosen Eltern über ein normales Hörvermögen verfügen. Allerdings fanden Schiff/Ventry $12 \%$ und Grüner $9 \%$ undiagnostizierte Hörstörungen. 20-25\% der Kinder waren bisher nicht audiometriert worden - eine Zahl, die angesichts der Risikogruppe irritiert $[3,10]$. Erst seit 2009 findet das Neugeborenenhörscreening als Regelleistung statt. Wahrscheinlich sinkt seitdem die Zahl der nicht entdeckten Hörauffälligkeiten auch bei Codas. 
Die Vermutung, dass Codas vermehrt Probleme in der auditiven Verarbeitung und Wahrnehmung durch mangelnde auditive Anregung in einem gehörlosen Elternhaus haben, hat sich nicht bestätigt [17].

\section{Merke}

Auf die Überprüfung des Hörens bei Codas muss geachtet werden.

\section{Dolmetschen}

Durch die häufig vorhandene Kompetenz von Codas sowohl Lautsprache als auch Gebärdensprache zu nutzen werden sie wie selbstverständlich als Dolmetscher zwischen ihren Eltern und der hörenden Umgebung betrachtet und auch eingesetzt. Sie fungieren als kulturelle Verbindung zwischen zwei relativ separierten Umgebungen. Es existiert eine große Bandbreite der Art zu dolmetschen. Verschiedene Qualitäten des Gebärdens, des Sprechens, des Absehens, der Pantomime und der Kombination aus allem werden verwendet. Einige Codas dolmetschen nur in bestimmten Situationen, z.B. beim Telefonieren. Sie dolmetschen aber auch in Situationen, die ihrem Alter und ihrer Kindesrolle nicht angemessen sind. Mädchen und Frauen dolmetschen am meisten, ein Ausdruck von traditionellen Rollenzuweisungen. Es hängt stark von der Quantität des Dolmetschens ab und ob Druck seitens der Eltern oder der hörenden Umgebung aufgebaut wird, ob das Dolmetschen als Überforderung empfunden wird. Problematisch wirkt sich unangemessenes Dolmetschen aus, wenn es zu einer Parentifizierung des Kindes führt, d.h. wenn das Kind die Rolle von Eltern, zumindest in Teilbereichen, übernimmt. Nicht wenige Codas sind jedoch stolz auf die besondere Fähigkeit der Bilingualität und des Dolmetschens. Viele professionelle Dolmetscher sind Codas [18]. In den letzten Jahren erfahren Codas Entlastung, indem vermehrt Dolmetscherdienste in Anspruch genommen werden können.

\section{Merke \\ Codas dolmetschen häufig zwischen Eltern und hörendem Umfeld. Sie werden manchmal überfor- dert durch eine zu große Häufigkeit, die Ausübung von Druck und nicht kindgemäße Situationen.}

\section{Interaktion und Kommunikation}

Wie schon in der Sprachentwicklung deutlich geworden, finden vielfältige Anpassungsprozesse zwischen Eltern und Kind statt, die Beziehung und Kommunikation bzw. Interaktion ermöglichen. Unterstützt wird dies durch das intuitive parenting in der frühen Kindheit und durch Lernprozesse, die auf gelingenden Erfahrungen basieren. Erwachsene passen sich unwillkürlich den Bedingungen des kleinen Kindes an und werden durch eine mehr oder weniger passende Antwort ermutigt. Von Beginn an kommunizieren die gehörlosen Mütter mit ihren hörenden Kindern so, dass sie in guter Beziehung und im Kontakt sein können. Sie gebrauchen häufiger ihre Stimme und sprechen bzw. nutzen Codeblending. Sie gebärden in kindgemäßer Weise, d.h. die Gebärden werden groß produziert, im Blickfeld des Kindes, öfter wiederholt. Mimik wird zunächst nicht als Bestandteil von Grammatik eingesetzt, sondern zum affektiven Ausdruck. Erst später, mit dem zunehmenden Gebrauch von Gebärdensprache, ändert sich dies. Die Kinder achten zunehmend auf die visuelle Zugänglichkeit ihrer Kommunikation, d.h. sie gehen ins Blickfeld der Mutter, winken oder berühren sie, um auf sich aufmerksam zu machen. Bereits im Alter von 19 Monaten wählen sie den Sprachmodus je nach Hörstatus des Gesprächspartners [19].

Allerdings zeigen Untersuchungen mit Vergleichsgruppen im Alter von 12 und 18 Monaten, dass Codas und ihre Mütter ähnliche Brüche in der Kommunikation und Interaktion erleben wie gehörlose Kinder und ihre hörenden Mütter. Feinfühligkeit in der Kommunikation und Interaktion gelingt am besten, wenn die Dyaden denselben Hörstatus haben [20]. Generell kann aber nicht von dauerhaften Beeinträchtigungen ausgegangen werden, insbesondere wenn die Mütter die Brüche heilen können. Jedoch muss besonderes Augenmerk auf das Gelingen der frühen Kindheit gelegt werden.

\section{Merke \\ Eltern und Kinder passen sich intuitiv an ihre spezifi- schen Bedingungen an. Trotzdem kommt es in der frühen Kindheit zu Brüchen in der Kommunikation.}

\section{Psychosoziale Entwicklung}

Die Situation „zwischen den beiden Welten“ hat ein Potenzial für Stress und Konflikt, aber auch für Wachstum und Stärke. Entscheidend für positive Auswirkungen sind empathische und responsible Bezugspersonen [2].

Codas werden oft sehr unterschiedliche, teilweise sich widersprechende Eigenschaften zugeschrieben. Sie seien empathisch, verantwortungsvoll, sozial kompetent und reflektierend. Sie sind stolz auf ihre Eltern und freuen sich über eigene Fähigkeiten wie z. B. bilingual zu sein oder zu dolmetschen. Sie seien aber auch von Schuldgefühlen ihren Eltern gegenüber geplagt, an übergroßer Verantwortung leidend, überfordert, Scham empfindend und von den Eltern auch im weiteren Sinne nicht verstanden. Alles das kann man in Biografien, psychoanalytischen Fallberichten oder entsprechenden Studien finden. 
FAZIT

Die besondere Situation von Codas ist von sehr unterschiedlichen Erwartungen, Normen und Werten bestimmt. Dieser Herausforderung begegnen Codas in der Regel mit Erfolg. Trotzdem kommt es zu Entwicklungen und Lebenssituationen, in denen sowohl die gehörlosen Eltern als auch die hörenden Kinder professionelle Unterstützung benötigen. Diese kann im sprachlichen als auch im psychosozialen Setting angesiedelt sein. Auf spezialisierte professionelle Unterstützungssysteme kann zurückgegriffen werden. Diese sind aber noch lange nicht in ausreichender Anzahl vorhanden. Eine gute Übersicht über die Angebote sowie eine ausführliche Bibliografie bietet die Homepage von „Leben auf dem Trapez“, einer Organisation von Fachleuten, die mit Codas und ihren Familien arbeiten. Codas selbst haben sich in CODA d.a.ch. zusammengeschlossen, um ihre einzigartige Lebenssituation zu bearbeiten.

Trotzdem bewältigen Codas offensichtlich überwiegend erfolgreich die besonderen Herausforderungen, in einem heterogenen Umfeld aufzuwachsen. Das Selbstwertgefühl und die Lebenszufriedenheit von erwachsenen Codas zeigen sich hinsichtlich Vergleichsgruppen nicht vermehrt beeinträchtigt. Gravierende psychosozialen Probleme sind nicht vorhanden.

\section{Merke}

Das Aufwachsen von Codas bietet offenbar genügend kompetenzstärkende und persönlichkeitsunterstützende Faktoren, um ein zufriedenes Leben führen zu können. Gehörlose Eltern verfügen mehrheitlich über entsprechende Fähigkeiten und auch das Umfeld, z. B. die hörenden Großeltern, tragen dazu bei. Dysfunktionale Familien mit entsprechenden Auswirkungen auf die kindliche Entwicklung finden sich nicht gehäuft.

\section{Interessenkonflikt}

Die Autorin gibt an, dass kein Interessenskonflikt besteht.

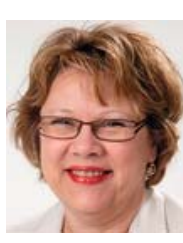 \\ Hiltrud Funk \\ Dr. phil. Hiltrud Funk studierte Schwerhöri- gen- und Sprachbehindertenpädagogik in Heidelberg und wurde 2004 promoviert (Das nicht gehörte Kind. Die Entfaltung des Selbst von hörenden Kindern mit hochgradig hör- behinderten Eltern. Möglichkeiten der Früh- förderung). Sie arbeitete als Sonderschulkonrektorin und Lei- terin der Frühberatungsstelle an der Schule am Sommerhoff- park, Frankfurt/M. Von 2005 bis 2014 leitete sie das Pfalz- institut für Hören und Kommunikation in Frankenthal und befindet sich jetzt im Ruhestand..}

\section{Korrespondenzadresse}

Dr. Hiltrud Funk

Hohenzollernstraße 28

67433 Neustadt an der Weinstraße

E-Mail: hiltrudfunk@web.de

\section{Literatur}

[1] Leonhardt A, Grüner B. Zur Sprachentwicklung hörender Kinder hörgeschädigter Eltern: 2. Ergebnisse einer Pilotstudie. Sprache - Stimme - Gehör 1997; 21: 69-73

[2] Funk H. Das nicht gehörte Kind. Die Entfaltung des Selbst von hörenden Kindern mit hochgradig hörbehinderten Eltern. Möglichkeiten der Frühförderung. Frankfurt a. Main: Brandes \& Apsel; 2004

[3] Grüner B. Die Sprachentwicklung hörender (Vorschul-)Kinder hochgradig hörgeschädigter bzw. gehörloser Eltern. Hamburg: Verlag Dr. Kovac; 2004

[4] Peter C, Raith-Kaudalka S, Scheithauer H. Gehörlose Eltern hörende Kinder. Coda Trainingsprogramm. Weinheim: Beltz Verlag; 2010

[5] Chilla S, Hofmann K. Entwicklungsaspekte und Erwerbsfaktoren im bimodal-bilingualen Erwerb von Codas (developmental and input aspects in bimodal-bilingual development of kids of deaf adults (kodas). DFGS Forum: Jahresschrift des deutschen Fachverbandes für Gehörlosen- und Schwerhörigenpädagogik 2014; 22: $73-89$

[6] Preston P. Mother Father Deaf. Living between sound and silence. Cambridge: Harvard University Press; 1995

[7] Meadow-Orlans KP, Spencer PE, Koester LS, eds. The world of deaf infants. A Longitudinal Study. Oxford: Oxford University Press; 2004

[8] Bishop M, Hicks S, eds. Hearing, Mother Father Deaf. Hearing people in deaf families. Washington: Gallaudet University Press; 2009

[9] Leigh IW, Marcus AL, Dobosh PK et al. Deaf/hearing cultural identity paradigms: Modification of the deaf identity development scale. Journal of Deaf Studies and Deaf Education 1998; $3: 329-338$

[10] Schiff N, Ventry ]. Communication problems in hearing children of deaf parents. Journal of Speech and Hearing Disorders 1976; 41: $348-358$ 
[11] Petitto LA, Holowka S, Sergio LE et al. Baby hands that move to the rhythm of language: hearing babies acquiring sign languages babble silently on the hands. Cognition 2003; 93: $43-73$

[12] Bonvillian JD, Orlansky MD, Folven RJ. Early sign language acquisition: Implications for theories of language acquisition. In: Volterra V, Erting CJ eds. From Gesture to Language in Hearing and Deaf Children. Berlin Heidelberg: Springer Verlag; 1990: 219-232

[13] Jones ML, Quigley SP. The acquisition of question formation in spoken English and American Sign Language by two hearing children of deaf parents. Journal of Speech and Hearing Disorders 1979; 2: $196-208$

[14] Johnson JM, Watkins RV, Rice ML. Bimodal bilingual language development in a hearing child of deaf parents. Applied Psycholinguistics 1992; 13: $31-52$

[15] van den Bogaerde B, Baker AE. Bimodal language acquisition in Kodas. In: Bishop M, Hicks SL, eds. Hearing, Mother Father Deaf. Washington: Gallaudet University Press; 2008: $99-131$

[16] Shook A, Marian V. Bimodal bilinguals co-activate both languages during spoken comprehension. Cognition 2012; 3: $314-324$
[17] Meilicke M]. Auditory processing in hearing children of deaf parents [Dissertation]. Graduate Faculty of the School of Human Behavior United States International University; 1993

[18] Preston P. Chameleon voices. Interpreting for deaf parents. Social Science and Medicine 1996; 12: 1681 - 1690

[19] Lillo-Martin D, de Quadros RM, Chen-Pichler D et al. Language choice in bimodal bilingual development. Frontiers in Psychology 2014: 10.3389/fpsyg.2014.01163 [published online]

[20] Meadow-Orlans KP. Effects of mother and infant hearing status on interaction at twelve and eighteen months. Journal of Deaf Studies and Deaf Education 1997; 2: 26-36

Bibliografie

DOI http://dx.doi.org/10.1055/s-0042-100690 Sprache · Stimme · Gehör 2017; 41: 19-24 (c) Georg Thieme Verlag KG Stuttgart · New York ISSN 0342-0477 